\title{
ROTATIONAL ANALYSIS OF THE RYDBERG SPECTRUM OF WATER
}

\author{
M. S. CHILD, $\dagger$ R. D. GILBERT $\dagger$ and Ch. JUNGEN§ \\ $\dagger$ Theoretical Chemistry Department, 5 South Parks Road, Oxford \\ $O X 13 U B, U K$ \\ $\ddagger$ Laboratoire Aimé Cotton, Université de Paris Sud, 91405 Orsay, France
}

\begin{abstract}
Two techniques for rotational analysis of the $n d \leftarrow 1 b_{1}$ Rydberg spectra of $\mathrm{H}_{2} \mathrm{O}$ and $\mathrm{D}_{2} \mathrm{O}$ are discussed-a Coriolis coupled asymmetric top model for the room temperature $3 d \leftarrow 1 b_{1}$ cluster, and an extension of multichannel quantum defect theory to asymmetric tops, which is applied to recent jet cooled photoionization spectra of the $n d \leftarrow 1 b_{1}$ series converging to the (100) vibrational levels of $\mathrm{H}_{2} \mathrm{O}^{+}$and $\mathrm{D}_{2} \mathrm{O}^{+}$. The quantum defects obtained by the two methods are in good agreement and new ionization limits to the (100) series are derived: $\mathrm{IP}(100)=104985(2) \mathrm{cm}^{-1}$ for $\mathrm{H}_{2} \mathrm{O}$ and $104262(1) \mathrm{cm}^{-1}$ for $\mathrm{D}_{2} \mathrm{O}$.
\end{abstract}

\section{INTRODUCTION}

This paper summarises recent work $^{1,2}$ on the rotational analysis of asymmetric top spectra in the Rydberg region, with particular reference to the strong $n d \leftarrow 1 b_{1}$ series of $\mathrm{H}_{2} \mathrm{O}$ and $\mathrm{D}_{2} \mathrm{O}^{3}$. The problems concern collapse of the electronic energy spacings as $n$ increases, leading to a transition from Hund's angular momentum coupling case (b) to Hund's case (d). Even at $n=3$ the room temperature absorption spectra $^{3,4}$ show distinct rotational profiles for only three of the four allowed electronic states, while the fifth $\bar{D}^{\prime \prime 1} A_{2}\left(3 d b_{2} \leftarrow 1 b_{1}\right)$ dark component has been observed by $3+1$ MPI. ${ }^{5}$ At higher energies the five electronic origins ultimately all lie within the span of the rotational constants and recent jet-cooled photoionization spectra ${ }^{6}$ of the series converging to the (100) vibrational levels of $\mathrm{H}_{2} \mathrm{O}^{+}$and $\mathrm{D}_{2} \mathrm{O}^{+}$show just two strong rotational lines which merge towards each other as $n$ increases.

Two methods of analysis are adopted. The first is a conventional Coriolis coupled asymmetric top treatment of the room temperature $3 d \leftarrow 1 b_{1}$ complex ${ }^{2}$ which was observed at high resolution in $1963^{4}$ but subsequently only partially analysed. ${ }^{7}$ Secondly the multichannel quantum defect theory of rotational channel interactions ${ }^{8}$ is extended to asymmetric tops ${ }^{1}$ and applied to analysis of the photoionization series. Notice that a similar quantum defect theory analysis of the $3 d$ complex, with separate rotational constants for the five electronic components would require an elaborate coupled vibrational-rotational channel treatment, of the kind so far attempted only for $\mathrm{H}_{2} \cdot{ }^{9}$ 


\section{A CORIOLIS COUPLED ASYMMETRIC TOP MODEL FOR THE $3 d$ COMPLEX}

To the extent that the dominant interactions occur within a given $n l$ complex the rotational structure may be modelled in terms of an upper state Hamiltonian of the form $^{1}$

$$
H=A\left(J_{a}-l_{a}\right)^{2}+B\left(J_{b}-l_{b}\right)^{2}+C\left(J_{c}-l_{c}\right)^{2}+X
$$

plus centrifugal stretching terms, where $\mathrm{A}, \mathrm{B}, \mathrm{C}$ and $\mathrm{X}$ are $(2 l+1) \times(2 l+1)$ diagonal matrices representing the rotational constants and electronic origins of the different $\lambda$ components of the complex. Written in this way, $H$ is diagonal in $J^{2}$ and $l^{2}$, the total and electronic angular momenta respectively, and diagonalization with respect to the body fixed projections $J_{a}$ and $l_{a}$ is conveniently performed in a basis of parity adapted symmetric top states $\left|\lambda K ; p_{e} p_{r}\right\rangle$ where $p_{e}$ and $p_{r}$ are parity labels with values 0 or 1 .

The forward calculation of a spectrum by means of Eq. (1) is quite straightforward, but problems of assignment of the $3 d \leftarrow 1 b_{1}$ complexes of $\mathrm{H}_{2} \mathrm{O}$ and $\mathrm{D}_{2} \mathrm{O}$, required for the reverse calculation, are complicated by strong Coriolis coupling, particularly between the ${ }^{1} A_{1}$ and ${ }^{1} B_{1} \tilde{F}$ state components whose origins lie within only $16 \mathrm{~cm}^{-1}$ for both $\mathrm{H}_{2} \mathrm{O}$ and $\mathrm{D}_{2} \mathrm{O}$. As a consequence the upper level of any asymmetry doublet in say the $\tilde{F}\left({ }^{1} A_{1}\right)$ component is so strongly coupled to the lower level of the $\tilde{F}\left({ }^{1} B_{1}\right)$ component that the resultant mixed states concentrate spectroscopic intensity into two of the four possible transitions. At the same time the effects of any asymmetry terms involving $(B-C)$ are quenched, so that the upper rotational states with $K^{\prime} \neq 0$ behave like symmetric tops, while those with $K^{\prime}=0$ (which are unaffected by the Coriolis terms) retain their asymmetric top character.

Another complication to the analysis is selective predissociation, which allows only $K^{\prime}=0,1$ branches in the $\tilde{E}$ and $\tilde{E}^{\prime}$ bands but $K^{\prime}$ up to 4 in the $\tilde{F}$ bands. There is

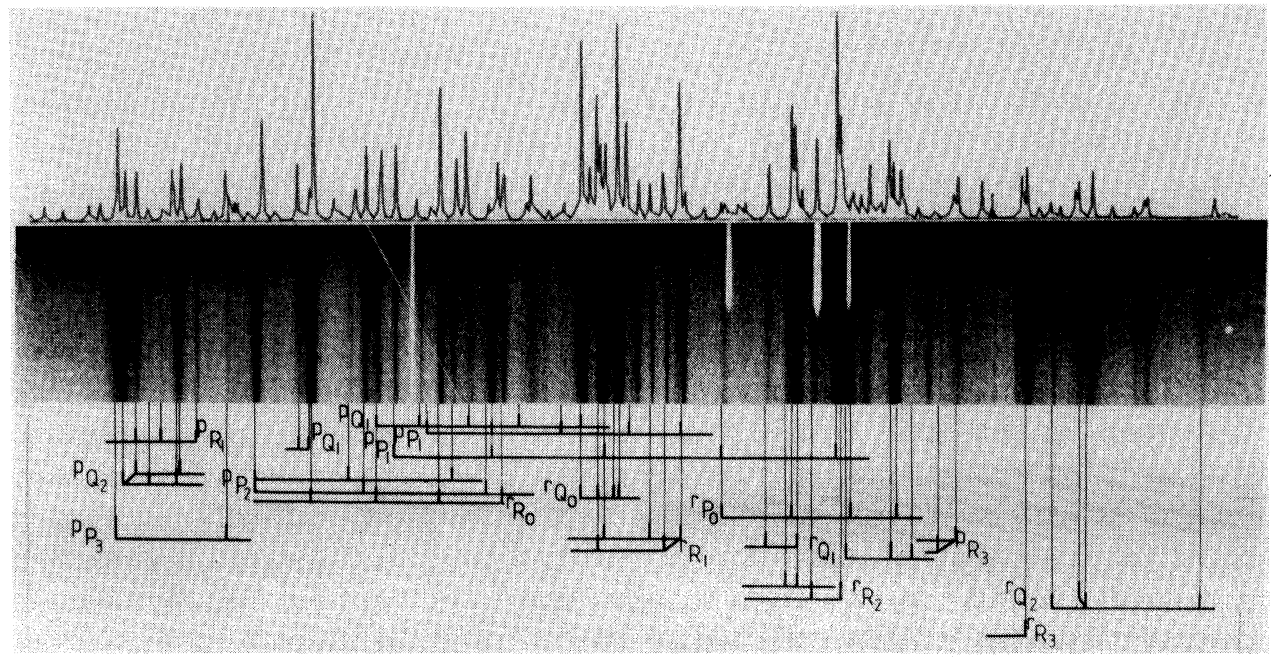

Figure 1 Assignment and simulation of the $\tilde{F}$ bands of $\mathrm{H}_{2} \mathrm{O}$. 
also a strong perturbation $\left(\simeq 150 \mathrm{~cm}^{-1}\right)$ of the $K^{\prime}=0$ levels of the $\tilde{E}^{\prime}$ state by a nearby linear $3 p b_{2} \leftarrow 3 a_{1}$ state.

Figure 1 gives a reconstruction of the $\tilde{F}$ band of $\mathrm{H}_{2} \mathrm{O}$ and Table 1 contains the derived spectroscopic parameters. ${ }^{2}$ Entries in brackets could not be determined by least squares optimisation, but values have been chosen to be consistent with reasonable geometries and inertial defects. Several of the latter are however sufficiently large to indicate the presence of interactions (perhaps with nearby $4 p$ states) that cannot be assessed without inclusion of additional data.

Table 1 Derived spectroscopic parameters

\begin{tabular}{|c|c|c|c|c|c|c|}
\hline \multirow[b]{2}{*}{$\mathrm{H}_{2} \mathrm{O}$} & \multicolumn{4}{|c|}{$3 d$ complex ${ }^{a}$} & \multicolumn{2}{|c|}{$n d \leftarrow 1 b_{1}$ series $^{b}$} \\
\hline & A $\mathrm{cm}^{-1}$ & B $\mathrm{cm}^{-1}$ & $\mathrm{C} \mathrm{cm}^{-1}$ & $\mathrm{X} \mathrm{cm}^{-1}$ & $\mu(\mathrm{n}=3)$ & $\mu(\mathrm{n}=6-10)$ \\
\hline $\begin{array}{l}\tilde{D}^{\prime 1} A_{2}\left(3 d b_{2}\right) \\
\tilde{E}^{1} B_{1}\left(3 d a_{1}\right) \\
\tilde{E}^{\prime 1} B_{2}\left(3 d a_{2}\right) \\
\tilde{F}\left\{\begin{array}{c}{ }^{1} A_{1}\left(3 d b_{1}\right) \\
{ }^{1} B_{1}\left(3 d a_{1}\right)\end{array}\right. \\
\text { ion }^{c}\end{array}$ & $\begin{array}{c}24.92 \\
36.12 \\
{[29.0]} \\
{[27.38]} \\
27.38 \\
29.026\end{array}$ & $\begin{array}{l}12.24 \\
12.50 \\
12.53 \\
11.61 \\
11.84 \\
12.422\end{array}$ & $\begin{array}{l}7.90 \\
8.91 \\
8.21 \\
8.69 \\
8.03 \\
8.469\end{array}$ & $\begin{array}{l}86189.0 \\
88502.9 \\
88927.9 \\
89679.7 \\
89696.3\end{array}$ & $\begin{array}{r}0.3463 \\
0.1243 \\
0.0771 \\
-0.0112 \\
-0.0145\end{array}$ & $\begin{array}{l}0.327 \\
0.155 \\
0.067 \\
0.013 \\
0.007\end{array}$ \\
\hline
\end{tabular}

$\mathrm{IP}\left(v_{1}=1\right) / \mathrm{cm}^{-1} 104985$

$\mathrm{IP}\left(v_{1}=0\right) / \mathrm{cm}^{-1} 101772$

\begin{tabular}{lclccrc}
$D_{2} O$ & $\mathrm{~A} \mathrm{~cm}$ & $\mathrm{~B} \mathrm{~cm}$ & $\mathrm{C} \mathrm{cm}$ & $\mathrm{X} \mathrm{cm}$ & $\mu(\mathrm{n}=3)$ & $\mu(\mathrm{n}=8-13)$ \\
\hline$\tilde{D}^{\prime \prime 1} A_{2}\left(3 d b_{2}\right)$ & 14.66 & 5.96 & 4.15 & 86138.0 & 0.3631 & {$[0.327]^{e}$} \\
$\tilde{E}^{1} B_{1}\left(3 d a_{1}\right)$ & 16.31 & 6.26 & 4.13 & 88686.1 & 0.1200 & {$[0.155]^{e}$} \\
$\tilde{E}^{\prime 1} B_{2}\left(3 d a_{2}\right)$ & {$[15.00]$} & 5.81 & {$[4.15]$} & 89090.4 & 0.0754 & {$[0.067]$} \\
$\tilde{F}\left\{{ }^{1} A_{1}\left(3 d b_{1}\right)\right.$ & {$[15.10]$} & 5.10 & 4.27 & 89826.9 & -0.0123 & {$[0.013]$} \\
${ }^{1} B_{1}\left(3 d a_{1}\right)$ & 15.11 & 6.24 & 4.10 & 89842.8 & -0.0143 & {$[0.007]$} \\
ion $^{c}$ & 16.0325 & 6.240 & 4.4066 & & &
\end{tabular}

$\mathrm{IP}\left(v_{1}=1\right) / \mathrm{cm}^{-1} 104262$

$\mathrm{IP}\left(v_{1}=0\right) / \mathrm{cm}^{-1} 101920^{b}$

${ }^{a}$ Coriolis coupled asymmetric top ${ }^{2} ;{ }^{b}$ Quantum defect theory ${ }^{1} ;{ }^{c}$ Lew $^{12} ;$ Lew and Groleau ${ }^{13} ;{ }^{d}$ assuming core stretching frequencies $v_{1}=3213.0 \mathrm{~cm}^{-1}$ and $2342 \mathrm{~cm}^{-1}$ for $\mathrm{H}_{2} \mathrm{O}^{+14}$ and $\mathrm{D}_{2} \mathrm{O}^{+15}$ respectively; ${ }^{e}$ assumed common for $\mathrm{H}_{2} \mathrm{O}$ and $\mathrm{D}_{2} \mathrm{O}$.

The resulting ordering of the five components confirms that first suggested by the Hartree-Fock calculations of Goddard and Hunt. ${ }^{10}$

\section{MULTICHANNEL QUANTUM DEFECT THEORY FOR ASYMMETRIC TOPS $^{2}$}

Multichannel quantum defect theory for rotational channel interactions has three ingredients. ${ }^{3}$ The quantum defects $\mu_{l \lambda}$ themselves translate into Born-Oppenheimerlike electronic origins

$$
T_{n l \lambda}=\mathrm{I}-\frac{R_{v}}{\left(n-\mu_{l \lambda}\right)^{2}}
$$


to which one would add asymmetric rotational eigenvalues $E_{J K}$ in performing a conventional case (b) isolated state rotational analysis. However in quantum defect theory the rotational energies are carried by the energies $E_{N^{+} K^{+}}$of the positive ion, which constitute separate ionization limits for the case (d) Rydberg series

$$
E_{n N K}=\mathrm{I}+E_{N^{+} K^{+}}-\frac{R_{y}}{n^{2}}
$$

where $N_{+}$, consistent with given $J$ and $l$, spans the range from $|J-l|$ to $J+l$. Finally there is a frame transformation $\left\langle\lambda K \mid N^{+} K^{+}\right\rangle$(which is diagonal in $J$ and $l$ ) which plays the same role in quantum defect theory as the inclusion of Coriolis terms in Eq. (1); when applied to Eq. (1) it would transform the rotational part of $H$ to the case (d) form

$$
H_{r o t}=A N_{a}^{+2}+B N_{b}^{+2}+C N_{c}^{+2}
$$

but make $\mathrm{X}$ non-diagonal.

Given these ingredients, the quantum defect equations, which determine the energy eigenvalues for a set of interacting closed channels, may be expressed as ${ }^{8}$

where

$$
\sum_{j}\left[K_{i j}+\tan \beta_{j}(E)\right] Z_{j}=0
$$

$$
\begin{aligned}
K_{i j} & =\sum_{\alpha}\langle i \mid \alpha\rangle \tan \pi \mu_{a}\langle\alpha \mid j\rangle \\
\beta_{j}(E) & =\pi\left\{R y /\left(E_{j}^{+}-E\right)\right\}^{1 / 2}
\end{aligned}
$$

in which $i$ and $j$ denote $N^{+} K^{+}$combinations and $\alpha$ is a short for $\lambda K$. Each half cycle of the term $\tan \beta_{j}(E)$ in Eq. (5) corresponds roughly to a new principal quantum number $n$ and the increasing rate of change of $\beta_{j}(E)$ as $E$ approaches $E_{j}^{+}$is responsible for the convergence of the various series. The coefficients $Z_{j}$ relate to amplitudes of different case (d) states $\left|N^{+} K^{+}\right\rangle$in the wavefunction.

To implement this theory for polyatomic molecules, Child and Jungen ${ }^{1}$ constructed the required frame transformation from the following matrix elements for symmetric tops, in which $p=\left(p_{e}+p_{r}\right) \bmod 2$

$$
\begin{aligned}
& \left\langle\lambda K ; p_{e} p_{r} \mid N^{+} K^{+} ; p\right\rangle=\frac{(-1)^{J}-K \sqrt{2 N^{+}+1}}{\left.\left[2\left(1+\delta_{\lambda O}\right)\left(1+\delta_{K O}\right) 1+\delta_{K^{+} O}\right)\right]^{1 / 2}}\left[\frac{1+(-1)^{p_{e}+p_{r}+p}}{2}\right] \\
& \times\left\{\left[\left(\begin{array}{rrr}
N^{+} & l & J \\
K^{+} & \lambda & -K
\end{array}\right)+(-1)^{p_{e}}\left[\begin{array}{rrr}
N^{+} & l & J \\
K^{+} & -\lambda & -K
\end{array}\right]\right.\right. \\
& \left.+(-)^{p+N^{+}+l}\left[\left(\begin{array}{rrr}
N^{+} & l & J \\
-K^{+} & \lambda & -K
\end{array}\right)+(-)^{p} \cdot\left(\begin{array}{rrr}
N^{+} & l & J \\
-K^{+} & -\lambda & -K
\end{array}\right)\right]\right\}
\end{aligned}
$$


and the following case (d) transition amplitudes

$$
\begin{aligned}
& \left\langle\lambda^{\prime \prime} K^{\prime \prime} ; p_{e}{ }^{\prime \prime} p_{r}{ }^{\prime \prime}|d| N^{+} K^{+} ; p^{\prime}\right\rangle \\
& =\frac{(-1)^{l^{\prime \prime}}+\lambda^{\prime \prime} 2 A \sqrt{2 N^{+}+1}}{\left[\left(1+\delta_{\lambda^{\prime \prime}}\right)\left(1+\delta_{K^{\prime} O}\right)\left(1+\delta_{K^{+} O}\right)\right]^{1 / 2}}\left[\frac{1+(-1)^{p^{e^{\prime \prime}+} p_{r^{\prime \prime}+p^{\prime}+1}}}{2}\right] \\
& \times\left(\begin{array}{lll}
l^{\prime} & 1 & l^{\prime \prime} \\
0 & 0 & 0
\end{array}\right)\left\{\begin{array}{lll}
J^{\prime \prime} & l^{\prime \prime} & N^{+} \\
l^{\prime} & J^{\prime} & 1
\end{array}\right\}\left\{\left[\left(\begin{array}{lll}
l^{\prime \prime} & N^{+} & J^{\prime \prime} \\
\lambda^{\prime \prime} & K^{+}- & -K^{\prime \prime}
\end{array}\right)+(-1)^{N^{+}+l^{\prime}+p^{\prime}}\left(\begin{array}{ccc}
l^{\prime \prime} & N^{+} & J^{\prime \prime} \\
\lambda^{\prime \prime} & -K^{+} & -K^{\prime \prime}
\end{array}\right)\right]\right. \\
& +(-1)_{e}^{p_{e}^{\prime \prime}}\left[\left(\begin{array}{cc}
l^{\prime \prime} N^{+} & K^{\prime \prime} \\
-\lambda^{\prime \prime} K^{+}-K^{\prime \prime}
\end{array}\right)+(-1)^{N^{+}+l^{\prime}+p^{\prime}}\left(\begin{array}{ccc}
l^{\prime \prime} & N^{+} & J^{\prime \prime} \\
-\lambda^{\prime \prime} & -K^{+} & -K^{\prime \prime}
\end{array}\right)\right]
\end{aligned}
$$

which carry the selection rules $\Delta l= \pm 1, p_{e}{ }^{\prime \prime}+p_{r}{ }^{\prime \prime}+p^{\prime}=$ odd, $N^{+}=\left|J^{\prime \prime}-l^{\prime \prime}\right|$ to $J^{\prime \prime}+l^{\prime \prime}$, and $K^{+}=\left|K^{\prime \prime} \pm \lambda^{\prime \prime}\right|$.

Figure 2 shows a comparison between the observed photoionization spectrum ${ }^{6}$ and a quantum defect simulation obtained by optimising the quantum defects and the ionization potential to the observed data. ${ }^{1} \mathrm{~A}$ temperature of $20 \mathrm{~K}$ was assumed and ortho para exchange during the cooling process was taken to be negligible. Under these conditions the dominant spectral features are attributable to transitions from the lowest ortho $\left(1_{01}\right)$ rotational level of the ground state which corresponds to the $\left|l^{\prime \prime} J^{\prime \prime} \lambda^{\prime \prime} K^{\prime \prime} ; p_{e}{ }^{\prime \prime} p_{r}{ }^{\prime \prime}\right\rangle=|1110 ; 00\rangle$ state, in the axis system chosen by Child and Jungen. ${ }^{1}$ Consequently Eq. (8) predicts dominant transitions to $\left|N^{+} K^{+} ; p^{\prime}\right\rangle=|21 ; 1\rangle$ and $|11 ; 1\rangle$ which corresponds to a type $c$ spectrum with members of the series converging to the $2_{11}$ and $1_{11}$ states of the positive ion, as suggested by Åsbrink and Rabalais. ${ }^{11}$ By contrast Page et al $^{6}{ }^{6}$ assumed type a selection rules, with selective predissociation to account for the simplicity of the spectrum. The greater complexity at $n=6$ is indicative of partial case (b) character which allows mixed a, b or c type selection rules according to the symmetry of the relevant $n d$ electronic component.

Optimised quantum defects and ionization energies, consistent with the simulation are listed in Table 1. Both the magnitudes of the defects and their orbital assignments are in good agreement with those derived from the $3 d$ complex.
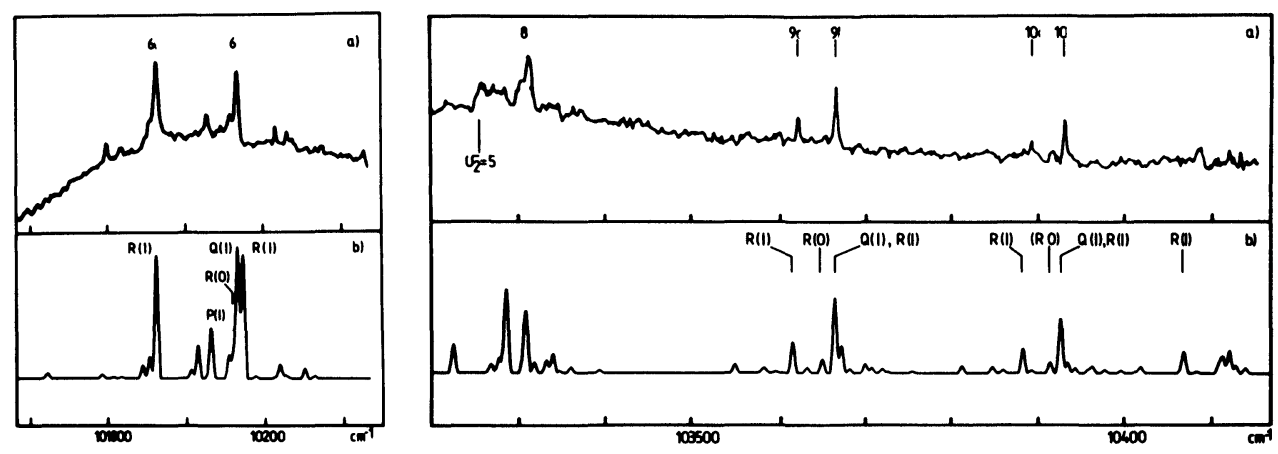

Figure 2 Observed and calculated photoionization series for $\mathrm{H}_{2} \mathrm{O}$. 


\section{References}

1. M. S. Child and Ch. Jungen, J. Chem. Phys. 93, 7756 (1990).

2. R. D. Gilbert, M. S. Child and J. W. C. Johns, Mol. Phys. (in press).

3. E. Ishiguro, M. Sasanuma, H. Masuko, T. Morioka and M. Nakamura, J. Phys. B11, 993 (1978).

4. J. W. C. Johns (private communication).

5. M. N. R. Ashfold, J. M. Bayley and R. N. Dixon, Can. J. Phys. 62, 1806 (1984).

6. R. H. Page, R. J. Larkin, Y. R. Shen and Y. T. Lee, J. Chem. Phys. 88, 2249 (1988).

7. M. N. R. Ashfold and J. M. Bayley, J. Chem. Soc. Faraday Trans. 86, 213 (1990).

8. C. H. Greene and Ch. Jungen, Adv. At. Mol. Phys. 21, 51 (1988).

9. Ch. Jungen and O. Atabek, J. Chem. Phys. 66, 5584 (1977).

10. W. A. Goddard and W. J. Hunt, Chem. Phys. Lett. 24, 464 (1974).

11. L. Åsbrink and J. W. Rabalais, Chem. Phys. Lett. 12, 182 (1971).

12. H. Lew, Can. J. Phys. 54, 2028 (1976).

13. H. Lew and R. Groleau, Can. J. Phys. 65, 739 (1987).

14. B. M. Dinelli, M. W. Crofton and T. Oka, J. Mol. Spectrosc. 127, 1 (1988).

15. J. E. Reutt, L. S. Wang, Y. T. Lee and D. A. Shirley, J. Chem. Phys. 85, 6928 (1986). 